\title{
DIVERSIDAD EN EL INSTRUMENTAL LÍTICO Y USO DEL ESPACIO \\ DURANTE EL HOLOCENO MEDIO Y TARDÍO EN LA COSTA NORTE DEL GOLFO SAN MATÍAS (RÍO NEGRO, ARGENTINA)
}

\author{
MARCELO CARDILLO* \& JIMENA ALBERTI*
}

\begin{abstract}
RESUMEN
La costa norte del golfo San Matías presenta una gran cantidad de loci arqueológicos, asignables a dos períodos diferentes del consumo de recursos marinos correspondientes al Holoceno medio-tardío y tardío final. Los conjuntos artefactuales líticos y las materias primas usadas en cada uno de estos bloques temporales presentan diferencias de índole cronológica y geográfica que son abordadas en el presente trabajo. A partir del análisis estadístico de los conjuntos recuperados, se establecen diferencias significativas en relación con la distribución de las clases de instrumentos en los bloques temporales definidos. Los resultados sugieren que la diversidad estaría estructurada cronológicamente más que en forma espacial, lo cual resulta acorde con el modelo de uso de los recursos propuesto previamente (Favier Dubois et al. 2009). Respecto del uso de materias primas líticas, se observan diferencias que tendrían que ver con cambios en las estrategias de aprovisionamiento a lo largo del tiempo.
\end{abstract}

PALABRAS CLAVE: Holoceno medio y tardío, diversidad y riqueza artefactual, materias primas líticas.

\section{LITHIC ARTIFACTS DIVERSITY AND SPACE USE DURING THE MIDDLE AND LATE HOLOCENE IN THE NORTHERN COAST OF SAN MATÍAS GULF (RÍO NEGRO, ARGENTINA)}

\section{ABSTRACT}

The Northern coast of San Matías Gulf presents a large number of archaeological loci, representative of two different time span within the model of space use and marine resources consumption proposed for the region (Favier Dubois et al. 2009). The lithic artifacts and the lithic raw materials that were used in each present chronological and geographic differences that are presented in this paper. Using statistical analysis, we have established significative differences related to the distribution of instrumental classes in the previously defined temporal blocks. The results suggest that diversity would have been structured

* IMHICIHU - CONICET. Saavedra 15 - 5to. piso. Buenos Aires (1083). marcelo.cardillo@gmail.com, jimealberti@gmail.com. 
chronologically rather than spatially which is consistent with the model of use of the resources previously proposed (Favier Dubois et al. 2009). In the use of lithic raw materials there are differences which would have to do with changes in strategies of provisioning during the occupation of the study area for the hunter-gatherers groups.

KEYWORDS: Middle and Late Holocene, artefactual richness and diversity, lithic raw materials.

\section{INTRODUCCIÓN}

En trabajos previos (Cardillo et al. 2007; Cardillo \& Scartascini 2007; Favier Dubois et al. 2009) se han observado diferencias en la distribución de la diversidad en los conjuntos líticos en el área de estudio. Estos resultados indican la existencia de loci con baja diversidad artefactual (principalmente instrumentos) y otros con una mayor preponderancia de desechos de talla, incluyendo las distintas categorías de lascas y núcleos. En algunos casos, dichas diferencias se relacionan directamente con el tamaño variable de las muestras recuperadas (Cardillo 2009).. Sin embargo, distintos sectores de la costa rionegrina muestran diferencias en la composición, riqueza artefactual y empleo de materias primas, independiente del tamaño de las muestras (Cardillo \& Scartascini 2011). Esto ha sido analizado paralelamente a otras líneas de evidencia, como la información cronológica, zooarqueológica y paleoambiental (Favier Dubois et al. 2008; Borella et al. 2007; Favier Dubois \& Borella 2007; Favier Dubois et al. 2009; Manzi et al. 2009). Los resultados obtenidos sugieren que el registro arqueológico en la escala regional se presenta distribuido de manera heterogénea, respondiendo a un uso variable del espacio a lo largo del tiempo que parece estar ligado, al menos en parte, a la explotación diferencial de recursos entre las costas norte y oste (Cardillo 2009; Favier Dubois et al. 2009; Cardillo \& Favier Dubois 2011; Cardillo \& Scartascini 2011). Uno de los aspectos más relevantes del registro arqueológico de la costa Norte es la presencia recurrente de gran densidad de restos de peces y de tecnología potencialmente asociada a su extracción al menos desde 6.000 años ${ }^{14} \mathrm{C}$ AP (Scartascini 2010). Esta evidencia de un uso temprano de los recursos marinos está acompañada de información isotópica proveniente de restos humanos (Favier Dubois et al. 2009) sobre la cual fue posible definir dos momentos en la ocupación, uso del espacio y explotación de recursos marinos en la costa (ver Tabla 1).

El primero de estos momentos comprendería el lapso que se ubica entre los 4.800 y los 2.200 años ${ }^{14} \mathrm{C}$ AP y habría implicado un uso intensivo de la costa, que dataría desde los 6.000 años ${ }^{14} \mathrm{C}$ AP (Favier Dubois \& Scartascini 2011). En estos momentos se habría dado el aprovechamiento de los recursos marinos, en particular peces (aunque también moluscos, mamíferos y aves marinas y crustáceos), tal como lo evidencia la aparición conspicua de pesas líticas de manufactura simple a lo largo de la costa del área estudiada (Favier Dubois et al. 2009; Scartascini 2010).

El segundo momento de ocupación abarca entre los 1.500 y los 450 años ${ }^{14} \mathrm{C}$ AP. Para estos momentos se registra una mayor presencia de fauna terrestre en los sitios (guanaco, Rheidae y pequeños mamíferos), que se suma al consumo de fauna marina que se verifica desde el bloque anterior (Favier Dubois et al. 2009; Marani \& Scartascini 2011). Algunos rasgos tecnológicos son conspicuos de este período, como la cerámica y el cambio en la morfología y tamaño de las puntas de proyectil (Favier Dubois et al. 2009), y la aparición de cáscaras de huevo grabadas (Fiore \& Borella 2010).

Por otro lado, la información cronológica y geomorfológica obtenida indica que los loci de la costa norte del Golfo tienden a distribuirse diferencialmente en el espacio en relación con su cronología relativa. Muchas de las formaciones costeras más recientes, coincidentes con la línea de costa actual (ver mapa), poseen loci fechados entre los 2.000 y 1.000 años ${ }^{14} \mathrm{C}$ AP, mientras que los conjuntos recuperados en paleoacantilados y terrazas marinas pertenecientes al Pleistoceno y Holoceno medio, han sido fechados en torno a los 6.000-3.000 años ${ }^{14} \mathrm{C}$ AP. El rango temporal observado en estos loci a partir de los fechados realizados es relativamente acotado, sugiriendo 
que la señal arqueológica de dichos sectores se conformó principalmente durante el rango cronológico mencionado. Esto podría relacionarse con el hecho que los loci quedaron emplazados en espacios con menor utilidad, por lo que fueron abandonados o utilizados de forma esporádica al acrecionar la costa, en algunos casos hasta dos $\mathrm{km}$.

A partir de la localización geomorfológica y cronológica (en los casos en los que ésta está disponible) es posible identificar sectores con potencial variable de descarte de artefactos a lo largo del tiempo, De esta manera, nos interesa caracterizar los conjuntos pertenecientes a estos sectores y a los diferentes bloques temporales antes descriptos en términos de su diversidad y distribución espacial de instrumentos líticos. Por lo tanto, el objetivo de este trabajo es explorar patrones en la diversidad en la tecnología lítica durante el Holoceno medio y tardío. Empleando procedimientos estadísticos, se explora la existencia de diferencias composicionales y de estructuración espacial en la distribución del registro arqueológico en la costa norte rionegrina (ver Fig. 1). Asimismo, se realiza una primera caracterización y análisis de la abundancia de los distintos tipos de roca empleados entre bloques temporales con el fin contrastar patrones observados previamente (ver Alberti 2010 para una discusión más detallada sobre este punto).

\section{ASPECTOS METODOLÓGICOS}

Los conjuntos analizados proceden de 30 loci distribuidos en la costa norte rionegrina (que abarca desde San Antonio Oeste hasta el balneario El Cóndor, desembocadura del río Negro en el océano), para los cuales se dispone de 28 fechados que permiten asignarlos a distintos momentos del Holoceno medio-tardío (Tabla 1). Las muestras analizadas en este trabajo fueron tomadas mediante cuadrículas de muestreo de tamaños variables

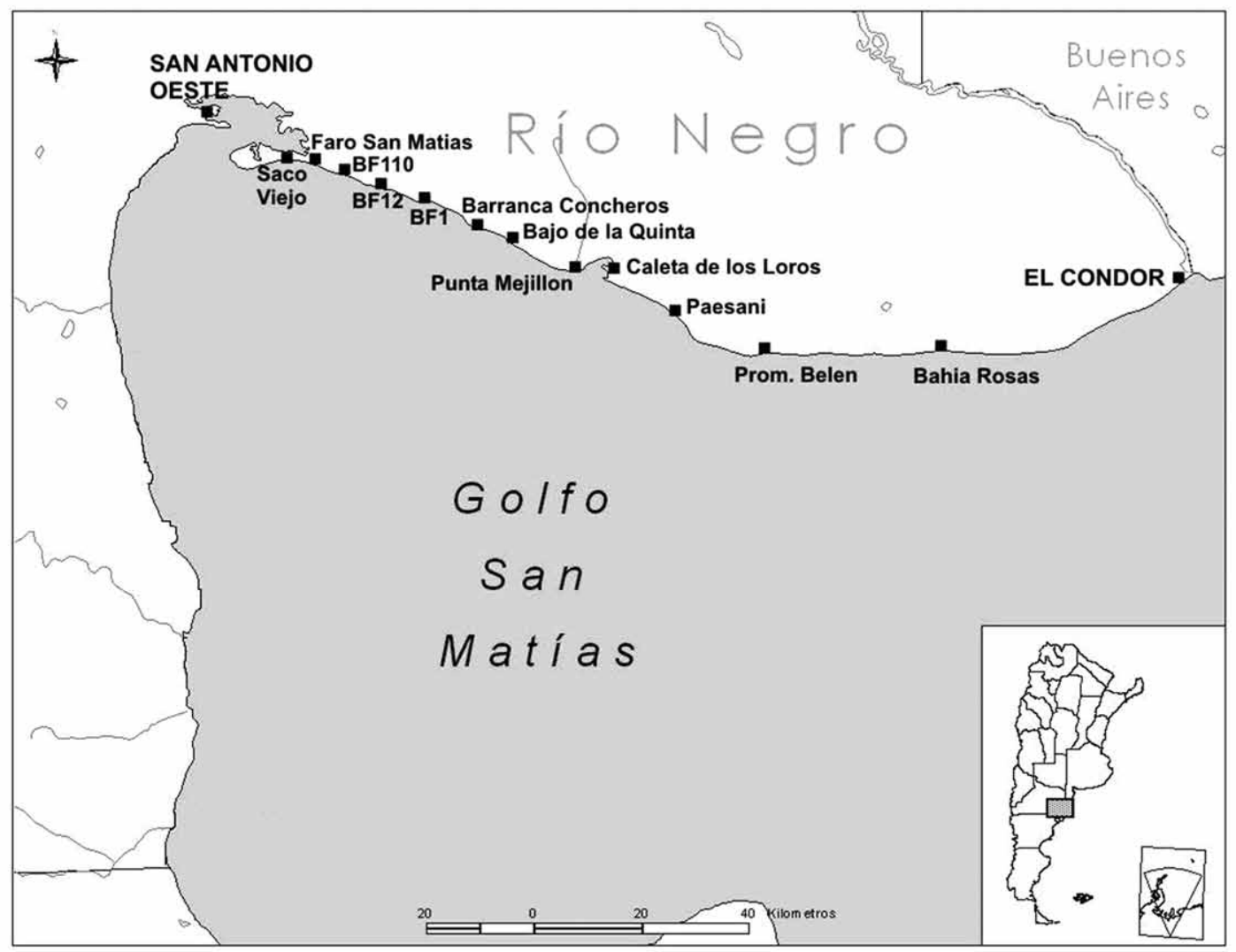

Fig. 1. Ubicación detallada de los loci arqueológicos (tomado y modificado de Favier Dubois et al. 2009) 
Tabla 1. Fechados de algunos loci de la costa norte del golfo San Matías, correspondientes a los dos bloques temporales de consumo de los recursos definidos para el área.

\begin{tabular}{|c|c|c|c|c|}
\hline \multicolumn{5}{|c|}{ BLOQUE ANTIGUO } \\
\hline Sitio arqueológico & Material datado & Edad & Lab. ref. & Cita \\
\hline $\begin{array}{l}\text { Bajo de la Quinta } \\
\text { Sector Otolitos }\end{array}$ & Otolitos corvina & $6080 \pm 80$ & LP-1904 & $\begin{array}{c}\text { Scartascini et al. } 2009, \\
\text { Favier Dubois y Kokot } 2011, \\
\text { Favier Dubois y Scartascini } 2011\end{array}$ \\
\hline Bahía Rosas 2 & Carbones & $3985 \pm 41$ & AA77298 & Cardillo 2009, Favier Dubois 2009 \\
\hline $\begin{array}{l}\text { Barranca de los } \\
\text { Concheros } 10\end{array}$ & Carbones & $2482 \pm 49$ & AA74748 & $\begin{array}{c}\text { Cardillo 2009, Favier Dubois 2009, } \\
\text { Favier Dubois y Kokot } 2011\end{array}$ \\
\hline \multicolumn{5}{|c|}{ BLOQUE RECIENTE } \\
\hline Sitio arqueológico & Material datado & Edad & Lab. ref. & Cita \\
\hline $\begin{array}{l}\text { Bajada de los } \\
\text { Pescadores }\end{array}$ & Carbones & $2197 \pm 38$ & AA81730 & Favier Dubois y Kokot 2011 \\
\hline Paesani - Sector 1 & Valvas & $1100 \pm 90$ & AC1710 & $\begin{array}{c}\text { Favier Dubois y Borella } 2007, \\
\text { Favier Dubois et al. } 2008\end{array}$ \\
\hline $\begin{array}{c}\text { Bajo de la Quinta - } \\
\text { La Noria oeste }\end{array}$ & Carbón & $450 \pm 80$ & LP-1926 & $\begin{array}{c}\text { Scartascini et al. } 2009, \\
\text { Favier Dubois y Kokot } 2011\end{array}$ \\
\hline
\end{tabular}

(2x2 m, 5x5 m o $10 \times 10 \mathrm{~m})$, situadas en puntos de mayor densidad de artefactos, los cuales en general se corresponden a loci definidos en superficies de deflación entre dunas (Favier Dubois et al. 2008). Se analizaron 298 instrumentos, considerando los que estuviesen enteros y los que presentasen fracturas menores que no impidiesen su asignación a una determinada clase. A fin de reducir la heterogeneidad interna debida a procesos aleatorios, como los vinculados al tamaño variable de las muestras analizadas (Cardillo 2009), no se tomaron en cuenta las clases de instrumentos presentes sólo una vez, como los morteros, las bolas y las puntas de proyectil.

La matriz original de frecuencia fue empleada en la estimación de la riqueza, medida como la cantidad de clases para cada loci, así como el índice de dominancia de Simpson (Magurran 2004). Este último, que mide el grado de concentración de cada conjunto, se aproxima a 1 cuando una sola clase está presente y a 0 cuanto mayor sea el número de clases en relación al tamaño de la muestra. Estos índices poseen un gran poder descriptivo aunque dependen de fluctuaciones en el tamaño de los conjuntos. Una relación significativa entre riqueza y tamaño de la muestra implica, por consiguiente, un sesgo que debe ser tomado en cuenta, especialmente dentro de un marco comparativo. En este caso, dada la relación observada entre diversidad y tamaño de la muestra analizada, se decidió estandarizar la muestra. Para la comparación entre conjuntos se empleó la proporción estimada a partir de la frecuencia de cada clase de artefacto en relación al tamaño total de la muestra por unidad de muestreo. Si bien esto no elimina totalmente el efecto del tamaño de las muestras, minimiza su efecto y permite la comparación directa (Legendre \& Gallagher 2001; Lepš \& Šmilauer 2003)

La matriz de clases fue empleada en métodos multivariados para explorar patrones en los datos y en el test de hipótesis entre bloques temporales. Con el fin de resumir la información de la proporción de clases se construyeron nuevas variables mediante escalamiento multidimensional no métrico (MDS). Este procedimiento es flexible ya que permite emplear distintos tipos de distancias y datos de abundancia, presencia o proporciones, mientras que minimiza la distancia entre los valores originales y los nuevos ejes. En particular se empleó el procedimiento de MDS disponible en el programa $\mathrm{R}$ (función metaMDS, R.2.12, 2005) que estandariza y re-escala los nuevos ejes de variación correspondientes a tipos de artefactos y casos, por lo que ambos pueden graficarse conjuntamente. Esto último facilita el análisis de los patrones resultantes.

Posteriormente las proporciones se emplearon en análisis discriminante basado en 
distancias (CAP) (Anderson \& Robinson 2003; Anderson \& Willis 2003; Anderson 2004). Este procedimiento permite encontrar los ejes de máxima separación en un conjunto de datos en donde previamente se han delimitado dos o más grupos. $\mathrm{Al}$ igual que su contraparte paramétrica, el CAP busca maximizar la separación entre grupos, aunque en este caso la probabilidad es establecida mediante permutaciones $(n=5000)$. En todos los casos, el nivel de significación empleado fue de $=0,05$. Este test posee la ventaja de realizarse mediante diferentes tipos de distancia. Para este análisis se emplearon distancias euclidianas ordinarias y de Bray-curtis; estas últimas toman en cuenta sólo la presencia conjunta, lo que puede ser conveniente en matrices con muchos "0" debidos a muestras pequeñas más que a características composicionales de los conjuntos (Legendre \& Legendre 1998). El análisis se acompañó de un test de dispersión o de varianza multivariada, con el fin de establecer si las diferencias observadas podrían estar vinculadas a diferencias en la varianza de los conjuntos o en su valor medio o centroide. En caso de poseer una dispersión semejante, las diferencias detectadas pueden atribuirse a la localización de los grupos, definida por su centroide, para este análisis de empleó el programa PERMDISP (Anderson 2004), utilizando el mismo número de permutaciones antes descrito.

Como este trabajo explora la estructura del registro instrumental, se empleó el test de Moran's I de autocorrelación espacial, ya que es esperable a lo largo del tiempo que sectores cercanos entre sí y que comparten las mismas propiedades ambientales, posean tasas de descarte artefactual similares.. Este test es análogo al análisis de correlación $r$ de Pearson (Legendre \& Fortin 1989) y otorga valores entre 1,0 y -1 . Valores positivos altos indican que los conjuntos tienden a poseer valores similares de la variable analizada a distintos rangos de distancia. Valores altos negativos significan, por el contrario, que dos conjuntos separados por una determinada distancia son muy diferentes entre sí. En tanto que aquellos valores cercanos a 0 indican autocorrelación nula y por consiguiente la ausencia de estructuración espacial. Las fluctuaciones gráficas en el valor de autocorrelación pueden ser luego interpretadas en forma de distintos patrones de estructuración espacial (ver Legendre y Fortin 1989; Legendre y Legendre 1998). Para aplicar este procedimiento se emplearon los valores de riqueza, dominancia, frecuencia absoluta de casos por loci y los dos primeros ejes de MDS. La probabilidad de autocorrelación significativa fue establecida mediante $(n=5000)$ permutaciones.

Por último, la frecuencia de las distintas materias primas, por instrumento, clasificadas macroscópicamente fue comparada para cada bloque temporal mediante el test de $X^{2}$, comúnmente empleado en variables categóricas. Dada la existencia de algunas frecuencias menores que $n=5$, se empleó también la estimación de probabilidad sugerida por Hammer \& Harper (2006) basada en procedimientos de aleatorización, implementada en el programa Past (2.08) (Hammer et al. 2001).

Los distintos procedimientos empleados aquí conforman herramientas para contrastar tres hipótesis de índole estadístico: una hipótesis de estructuración espacial sincrónica, sin tener en cuenta los bloques temporales (test de Moran) y otra hipótesis de estructuración temporal (análisis discriminante entre bloques temporales), tanto en las clases de artefactos como en la frecuencia de materias primas. La hipótesis nula de la primera hipótesis $\left(\mathrm{H}_{01}\right)$ asume una tasa de descarte de instrumentos homogénea a lo largo del espacio para todo el Holoceno mediotardío y reciente. La segunda hipótesis nula $\left(\mathrm{H}_{02}\right)$ propone que la composición instrumental, medida como proporciones de instrumentos dentro de la muestra, es la misma en los dos bloques temporales estudiados. En cuanto a las materias primas la $\mathrm{H}_{03}$ sostiene que no existen diferencias significativas en la frecuencia de rocas empleadas para confeccionar instrumentos entre bloques temporales.

\section{ANÁLISIS Y RESULTADOS}

La frecuencia de instrumentos entre los loci oscila entre 2 y 31, lo cual, inevitablemente, afecta la riqueza instrumental presente en los loci más pequeños ya que es más probable que efectos aleatorios afecten la presencia de instrumentos y por consiguiente, las clases representadas. Asimismo, la riqueza oscila entre 1 y 9 clases por loci, lo que también indica diferencias composicionales.

La correlación entre riqueza instrumental y el tamaño de la muestra en cada locus es alta y significativa $(R h o=0,88, p<0,05)$, lo cual significa que la riqueza observada está condicionada primariamente por el tamaño de la muestra. Esta correlación afecta 
potencialmente a todos los índices de diversidad que utilizan la riqueza como estimador. Por otro lado, los índices de dominancia oscilan entre 0,15 y 1 por lo que hay conjuntos compuestos por un solo tipo de instrumento, $y$ otros con valores de dominancia bajos, con una alta homogeneidad en la frecuencia representada de distintas clases. La dominancia media para el bloque temprano es de 0,30, con un desvío estándar de 0,12, mientras que para el bloque tardío la media es de 0,42 y el desvío estándar de 0,31, lo que indica dominancia y varianza mayores para este último. Sin embargo, tal como se observa en relación a la riqueza, este índice está correlacionado con el tamaño de la muestra (Dominancia Rho=$0,63, p<0,05)$. En el primer caso los resultados indican que las muestras de mayor tamaño poseen una distribución más regular de clases y por lo tanto una dominancia menor. Dada esta relación entre los estimadores de diversidad y el tamaño de la muestra, la interpretación directa de estos resultados está parcialmente sesgada. Por ello, los valores de riqueza se estandarizaron a proporciones en relación al tamaño de su correspondiente conjunto, lo que reduce el efecto del tamaño de las muestras y facilita las comparaciones.

Los instrumentos que se encuentran en mayor proporción en la muestra analizada son los denticulados ( $n=63,22 \%)$, seguidos por raspadores ( $n=44,15 \%)$, instrumentos de formatización sumaria y filos naturales con rastros complementarios $(n=38$, $13 \%$ y $n=34,12 \%$ respectivamente) (Fig. 2). Las restantes categorías se encuentran en proporción menor al 0,05 o 5\% del total (ver Tabla 2).

La proporción de instrumentos para los 30 loci analizados fue empleada posteriormente en análisis de escalamiento multidimensional (Fig. 3).

En los resultados del MDS (Fig.3), se observa que los casos pertenecientes al bloque temprano (cuadrados) sólo se solapan de forma parcial con aquellos pertenecientes al bloque tardío (cruces). Otro aspecto interesante es que éstos últimos parecen mostrar una mayor dispersión o variabilidad que los primeros, un fenómeno que se había observado en otras oportunidades (Cardillo 2009) y que no parece relacionarse simplemente con el tamaño de las muestras. En este caso, esto se debe a que la variabilidad interna de los conjuntos pertenecientes al bloque tardío (ver supra) es mayor. También se observa

Tabla 2. Porcentaje de instrumentos discriminados por bloques temporales respecto de la muestra total. FNCRC: Filo natural con rastros complementarios; Form. Sum: Instrumento de formatización sumaria; Pref, pta. proy: Preforma de punta de proyectil; Pref. bif.: preforma bifacial no determinada; Pta. bte: Punta burilante; Pta. proy: Punta de proyectil; Ret. sumario: Retoque sumario.

\begin{tabular}{|l|c|c|c|c|c|c|}
\hline \multicolumn{1}{|c|}{ Instrumento } & $\begin{array}{c}\text { Bloque } \\
\text { temprano }\end{array}$ & $\%$ & Bloque tardío & $\%$ & $\begin{array}{c}N \\
\text { total }\end{array}$ & $\begin{array}{c}\% \\
\text { total }\end{array}$ \\
\hline Bifaz & 4 & 2,08 & 1 & 0,94 & 5 & 1,68 \\
\hline Cepillo & 1 & 0,52 & 2 & 1,88 & 3 & 1,01 \\
\hline Chopper & 8 & 4,16 & 0 & 0 & 8 & 2,68 \\
\hline Denticulado & 54 & 28,12 & 9 & 8,49 & 63 & 21,14 \\
\hline FNCRC & 16 & 8,33 & 18 & 16,98 & 34 & 11,41 \\
\hline Form. sum. & 19 & 9,89 & 19 & 17,92 & 38 & 12,75 \\
\hline Muesca & 7 & 3,64 & 19 & 17,92 & 26 & 8,72 \\
\hline Percutor & 4 & 2,08 & 5 & 4,71 & 9 & 3,02 \\
\hline Pesa & 11 & 5,72 & 1 & 0,94 & 12 & 4,03 \\
\hline Pref. pta. proy. & 1 & 0,52 & 1 & 0,94 & 2 & 0,67 \\
\hline Pref. bif. & 7 & 3,64 & 8 & 7,54 & 15 & 5,03 \\
\hline Pta.bte. & 8 & 4,16 & 2 & 1,88 & 10 & 3,36 \\
\hline Pta. proy. & 1 & 0,52 & 1 & 0,94 & 2 & 0,67 \\
\hline Raedera & 10 & 5,20 & 5 & 4,71 & 15 & 5,03 \\
\hline Raspador & 29 & 15,10 & 15 & 14,15 & 44 & 14,77 \\
\hline Ret. sumario & 12 & 6,25 & 0 & 0 & 12 & 4,03 \\
\hline & 192 & 100 & 106 & 100 & 298 & 100 \\
\hline
\end{tabular}



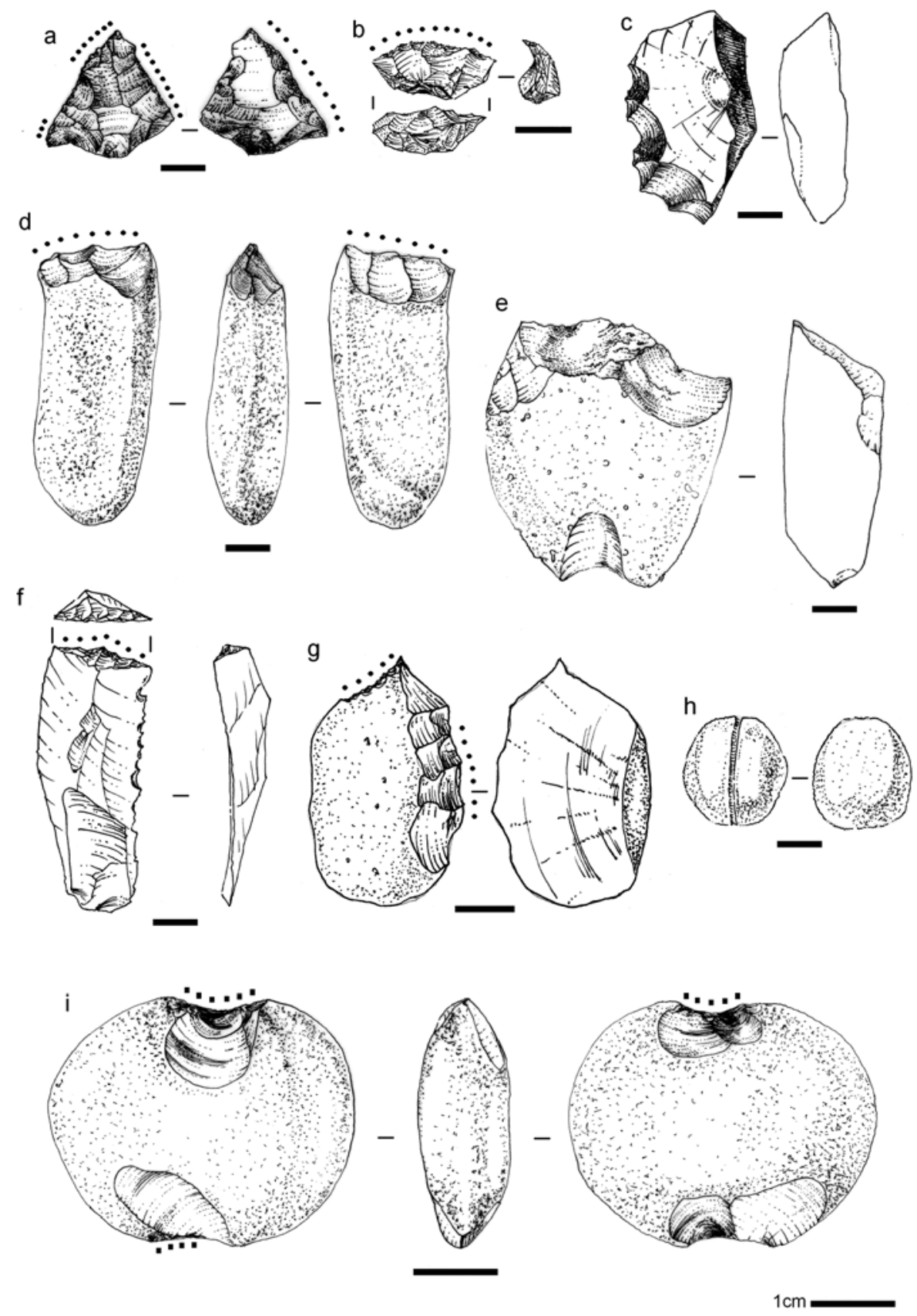

Fig. 2. Instrumentos recuperados en los distintos loci. a) Punta de proyectil retomada, calcedonia. b) Raspador, sílice. c) Denticulado, RGFO. d) Chopper, riolita. e) Pesa lítica retomada como chopper, RGFO. f) Raspador, sílice. g) Punta burilante y raedera, RGFO. h) Pequeña bola con surco, roca carbonática. i) Pesa lítica, RGFO. $\square$ rastros complementarios 
que algunas clases de instrumentos se distribuyen diferencialmente en el espacio correspondiente a uno u otro bloque. Para contrastar este patrón se realizó un análisis discriminante empleando la proporción de instrumentos sobre las muestras divididas en dos bloques temporales, dejando de lado la muestra perteneciente a Laguna de las Máquinas, de asignación temporal incierta al momento. Este análisis señala resultados significativos independientemente de la distancia empleada: euclidiana $=0,47, p<0,01$; BrayCurtis $=0,55, p<0,01$. Se observa que esta última medida de distancia, que toma en cuenta sólo la presencia conjunta de casos, capta una variación relativamente mayor que la distancia euclidiana. Sin embargo, la performance de ambos test es muy similar, ya que el porcentaje de discriminación correcta es del 77\% en ambos casos, por lo que ambos conjuntos comparten un 33\% de similitud. Las clases de instrumentos con mayor peso para separar ambos grupos son aquellas que poseen una mayor correlación con eje discriminante (Tabla 3).

Paralelamente se realizó un análisis de

Tabla 3. Correlación entre cada una de las clases y el eje discriminante para cada una de las distancias. Las correlaciones más altas (negativas o positivas) indican que dichas clases poseen un mayor rol en la separación entre bloques temporales (en negrita).

FNCRC: Filo natural con rastros complementarios; Form. sum.: Instrumento de formatización sumaria; Pref, pta. proy: Preforma de punta de proyectil; Pref. bif: preforma bifacial no determinada; Pta. bte: Punta burilante; Pta. proy: Punta de proyectil; Ret. sumario: Retoque sumario.

\begin{tabular}{|l|c|c|}
\hline & Bray-Curtis & Euclidiana \\
\hline Bifaz & $-0,2088$ & $-0,1275$ \\
\hline Cepillo & 0,2161 & 0,0918 \\
\hline Chopper & $-0,3481$ & $-0,3006$ \\
\hline Denticulado & $-0,6499$ & $-0,7292$ \\
\hline FNCRC & 0,2095 & 0,2577 \\
\hline Form. sum & 0,3726 & 0,3499 \\
\hline Muesca & 0,6883 & 0,7319 \\
\hline Percutor & 0,2955 & 0,1103 \\
\hline Pesa & $-0,5459$ & $-0,5712$ \\
\hline Pref pta. proy. & $-0,0686$ & 0,0253 \\
\hline Pref. bif. & $-0,1225$ & $-0,0395$ \\
\hline Pta_bte. & $-0,1906$ & $-0,2486$ \\
\hline Pta. proy & 0,1096 & 0,0036 \\
\hline Raedera & $-0,2871$ & $-0,1312$ \\
\hline Raspador & 0,1921 & 0,1919 \\
\hline Ret. sumario & $-0,3117$ & $-0,1878$ \\
\hline
\end{tabular}

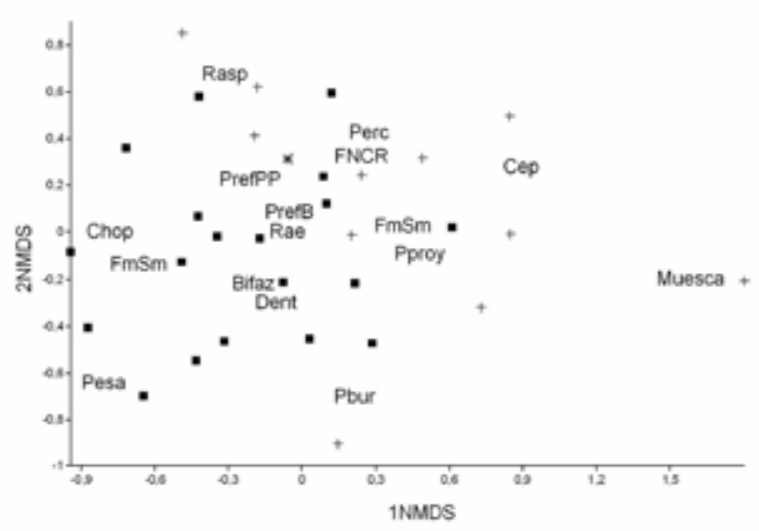

Fig. 3. Primeros ejes de escalamiento multidimensional. Loci bloque temprano. + Loci bloque tardío. . Sin cronología. Dent: denticulado; Prefpp: preforma de punta de proyectil; Proy.: punta de proyectil; FmSm: instrumento de formatización sumaria; Pbur: punta burilante; Perc:percutor; Cep: cepillo; FNCR: filo natural con rastros complementarios; Chop: chopper; Rae: raedera; Pref.: preforma bifacial no determinada; Rasp: raspador.

dispersión multivariado empleando las mismas distancias. Los resultados en ambos casos no son significativos (Bray-curtis $F=0,25, p>0,05$; Euclidiana $\mathrm{F}=0,68 \mathrm{p}>0,05)$. Esto quiere decir que las diferencias observadas no se relacionan con la varianza de los conjuntos, sino más bien con su valor centroide. Los resultados indican que la proporción de denticulados, muescas, instrumentos de retoque sumario y pesas son los que poseen un mayor peso en la discriminación entre bloques temporales, independientemente de la medida de distancia que se emplee. Pesas, denticulados y choppers son más frecuentes en el bloque temprano, mientras que las muescas e instrumentos de formatización sumaria lo son en el tardío.

Para explorar en qué medida la distancia entre las localidades afecta los valores de diversidad, se realizó el test de Moran de autocorrelacion espacial entre la distancia en metros entre loci y la frecuencia absoluta de instrumentos en cada uno de ellos, riqueza, índice de Dominancia y los dos primeros ejes de MDS. Los resultados indican que no existe una estructuración espacial a nivel global en los datos para las variables analizadas. En sólo dos variables (Fig. 4) se observaron valores significativos en intervalos de distancia de $30-40 \mathrm{~km}$, para el caso del primer eje de MDS, y de $15 \mathrm{~km}$, para el 
Tabla 4. Frecuencia y porcentaje de materias primas empleadas en la confección de instrumentos en los loci para cada bloque temporal.

\begin{tabular}{|c|c|c|c|c|c|}
\hline Tipo de roca & Bloque temprano & $\%$ & Bloque tardío & $\%$ & Total \\
\hline Andesita & 2 & 1,05 & 0 & 0 & 2 \\
\hline Criptocristalinas & 51 & 26,8 & 45 & 41,67 & 96 \\
\hline Dacita & 2 & 1,05 & 2 & 1,85 & 4 \\
\hline Pórfidos & 19 & 10 & 0 & 0 & 19 \\
\hline RGFO & 71 & 37,4 & 41 & 37,96 & 112 \\
\hline Riolita & 5 & 2,63 & 1 & 0,93 & 6 \\
\hline Sedimentarias & 1 & 0,53 & 0 & 0 & 1 \\
\hline Sílice & 39 & 20,5 & 19 & 17,59 & 58 \\
\hline Total & 190 & 100 & 108 & 100 & 298 \\
\hline
\end{tabular}

segundo. Estos resultados sugieren una estructura espacial del registro relativamente homogénea, con un tendencia baja a la formación de parches con valores locales de riqueza o diversidad diferentes en los intervalos de distancia antes mencionados, lo que puede deberse a distintas causas (ver discusión).

\section{MATERIAS PRIMAS}

Respecto de las materias primas sobre las que están formatizados los diferentes instrumentos que componen la muestra analizada, presentaremos sólo un análisis general a nivel descriptivo $\mathrm{y}$, en menor medida comparativo, a nivel de ambos

a
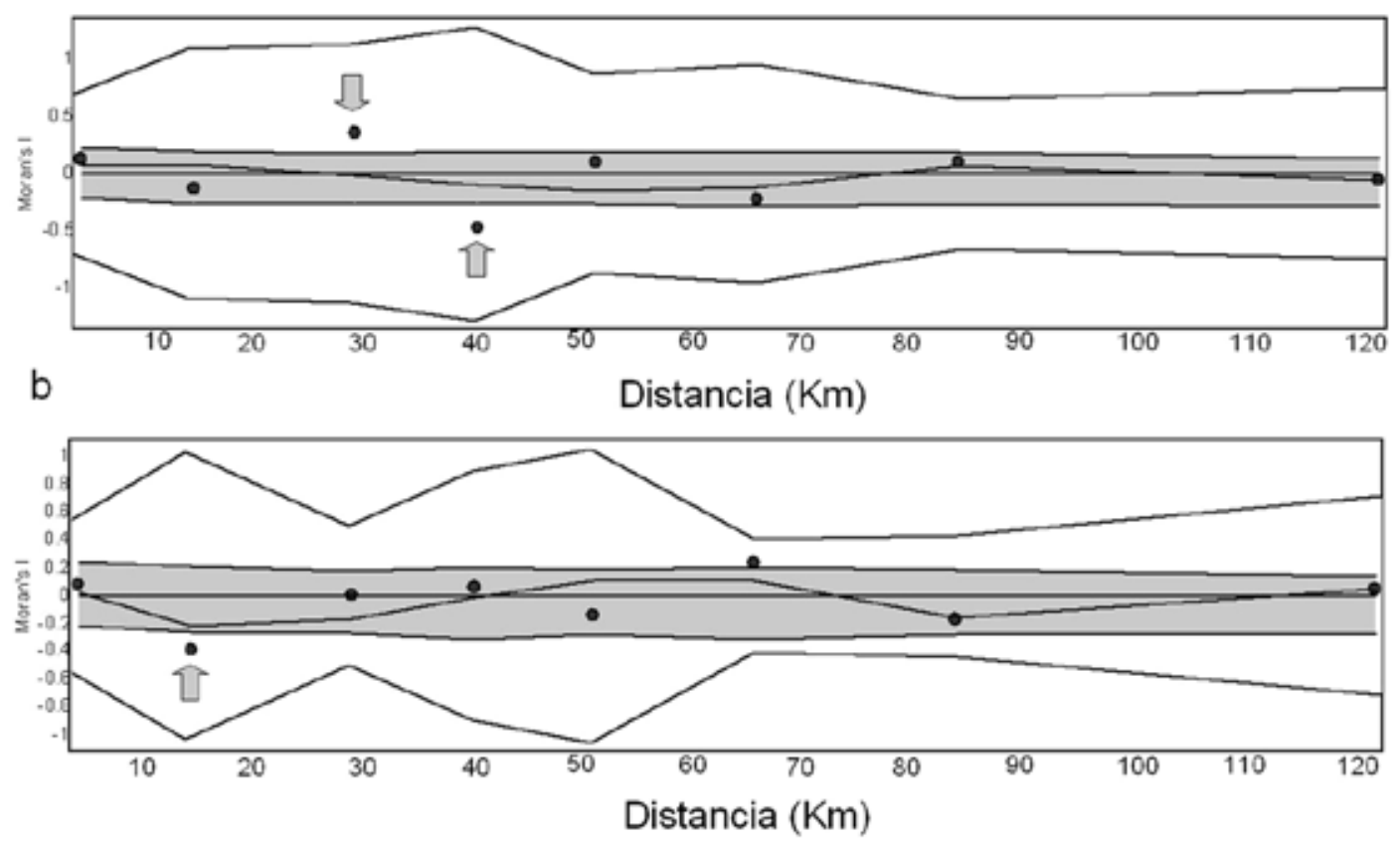

Fig. 4. Diagrama de autocorrelación a distintos intervalos de distancia (eje de las abscisas) medidos a partir del primer (a) y segundo (b) eje de escalamiento multidimensional. En gris se indica el intervalo del 95\% de confianza global para el test de Moran. La flecha señala el intervalo de distancia para el cual se encontró un patrón significativo. 
bloques temporales.

Tanto en el bloque temprano como en el tardío, las rocas más frecuentemente usadas fueron las de grano fino oscuro (RGFO: vitrófiros dacíticos, grauvacas, lutitas y ftanitas tipos que no pueden ser distinguidos macroscópicamente) (sensu Charlin 2005, 2009), seguidas de las rocas criptocristalinas (categoría que incluye calcedonias y jaspes, y mineraloides como los ópalos). En el caso de las primeras, en ambos bloques se utilizaron en proporciones similares pese a las diferencias en los tamaños de las muestras (Tabla 4). Sin embargo, en el caso de las rocas criptocristalinas su proporción es mayor en la muestra del bloque más tardío.

Otras variedades de rocas que se encuentran en proporciones menores, parecen distribuirse de forma desigual entre bloques temporales. Este es el caso de las rocas porfídicas, que componen el 10\% de la muestra del bloque temprano y no están presentes en las del tardío. Un patrón similar se repite en otras rocas de menor calidad para la talla andesitas, riolitas y sedimentarias más abundantes en el mencionado bloque, aunque en una proporción relativa muy baja. Estas diferencias son significativas en el test de $X^{2}$ $: 18,836 \mathrm{p}=0,008$, Monte Carlo $\mathrm{p}=0,004$ (Tabla 4). En términos generales, el bloque temprano posee una mayor riqueza de clases de materias primas $(n=8)$ que el tardío $(n=5)$ aunque esto último, al igual que en el caso de los instrumentos, podría relacionarse con diferencias en el tamaño de la muestra (ver Cardillo \& Scartascini 2007; Alberti 2010).

\section{DISCUSIÓN Y CONCLUSIONES}

Los análisis realizados muestran que si bien la riqueza observada está mediada por la diversidad (y, por consiguiente, los índices basados sobre esta variable están sesgados), existen diferencias significativas en la proporción en la que algunas de las clases de instrumentos recuperadas se distribuyen entre los bloques temporales previamente definidos (ver supra). Instrumentos como pesas, choppers y denticulados son más frecuentes en el bloque temprano, mientras que raspadores, instrumentos de retoque sumario y muescas lo son en el tardío. Estas diferencias en la tasa de descarte de determinadas clases podrían relacionarse con variaciones en el modo de uso de determinados espacios a lo largo del tiempo, lo cual podría vincularse con los cambios registrados en las estrategias de explotación y consumo de recursos marinos y terrestres. Esto último está sustentado por otras líneas de evidencia, como la arqueofaunística y la de los isótopos estables sobre restos humanos, que sugieren fluctuaciones en la dieta de las poblaciones humanas en la región, especialmente en cuanto al rol de los recursos marinos (Favier Dubois et al. 2009; Scartascini 2010; Marani y Scartascini 2011). Los análisis de autocorrelación espacial señalan que para todo el espacio analizado no se identifica un patrón que indique diferencias significativas en la diversidad de los conjuntos líticos en sectores particulares de la franja costera. Por el contrario, las diferencias observadas en forma de oscilaciones en escalas espaciales pequeñas parecen vincularse a conjuntos que pertenecen a bloques temporales distintos. Esto implica, entre otras cosas, que conjuntos que se encuentran espacialmente cercanos pueden poseer una composición instrumental muy diferente. Esto es relevante a la luz de la cercanía espacial entre los conjuntos de los bloques temprano y tardío que en ocasiones, como es el caso de Bahía Final, es de cientos de metros. En escalas espaciales algo mayores, se observó, una tendencia a la formación de parches que podría darse para los intervalos de 15-35-40 km (Fig. 4). Al ver la distribución de los loci se observa que esto puede deberse a que los del bloque temprano tienden a acumularse en algunos sectores, como el interior de la bahía San Antonio, el Bajo de la Quinta y la bahía Creek, mientras que la mayoría de los del bloque tardío se distribuyen en la línea costera actual o próximos a ella, especialmente en el sector de Bahía Final, Faro San Matías y Saco Viejo y Punta Villarino (ver Fig. 1).

En conjunto, la estadística espacial junto con los resultados del test discriminante entre bloques temporales, sugiere que la diversidad instrumental no está estructurada geográficamente sino más bien cronológicamente. Es decir, conjuntos con similar cronología tienden a estar compuestos por las mismas proporciones de instrumentos, independientemente de su localización o cercanía espacial. Esto podría significar que el espacio costero fue utilizado de una manera relativamente homogénea dentro de los distintos bloques temporales. En cuanto al empleo de materias primas, se observaron diferencias significativas entre bloques cronológicos, particularmente en la proporción de rocas criptocristalinas, más utilizadas 
en el bloque tardío. Esto contradice formulaciones previas basadas en datos de una sola localidad (Alberti 2010) y deja ver que los resultados obtenidos no concuerdan con lo esperado para un contexto de uso inicial del espacio con mayor movilidad y equipamiento personal (sensu Kuhn 2004). Más bien, la mayor proporción de rocas de calidad inferior para la talla, en particular las rocas porfídicas, sugiere una menor selectividad en cuanto a la calidad de la materia prima para el bloque temprano, ya que hemos visto que estas rocas se emplearon para confeccionar diferentes tipos de instrumentos (principalmente pesas de red y choppers, pero también raederas y denticulados, que aparecen en los sitios junto con núcleos de este tipo de rocas) (Alberti 2010). Para el bloque tardío, la evidencia del análisis artefactual sugiere un incremento en la tasa de descarte de desechos de talla y nódulos de calidad buena y muy buena para la talla (Cardillo \& Scartascini 2010; Cardillo \& Favier Dubois 2011). Por otro lado, algo similar puede ocurrir con los morteros, que podrían indicar estrategias de equipamiento de algunos espacios, aunque aún no se ha estudiado detalladamente su distribución espacial y cronológica. Es posible, entonces, que una mayor proporción en el uso de rocas criptocristalinas se relacione con una disminución del costo de aprovisionamiento a partir de una estrategia mantenida en el tiempo de transporte de estas materias primas a los loci. De ser así, sería esperable que no se observen diferencias en la intensidad de uso de estas rocas a partir del análisis de otros indicadores, como núcleos y lascas. Por consiguiente, este patrón debe ser explorado en mayor detalle incorporando distintos subproductos de la talla lítica, así como información más detallada de la oferta de materias primas en el área.

Por otro lado, un uso combinado de distintos índices (sensu Shott 2010) junto con un relevamiento detallado de la densidad del registro lítico para cada bloque temporal puede ser incorporado para contratar las hipótesis generadas en este trabajo. Entendidas de este modo, las diferencias observadas en la riqueza pueden ser una función de la intensidad de ocupación de los loci en los distintos bloques temporales. Una estrategia en la que se está trabajando es en el muestreo sin recolección de los conjuntos superficiales, modalidad que permite obtener información minimizando el impacto arqueológico, lo cual es especialmente importante en los sectores de baja densidad artefactual que caracterizan al bloque temprano. El relevamiento de nuevos loci y la incorporación de los desechos de talla al análisis permitirá ampliar el tamaño de los conjuntos y evaluar el rol de nuevas variables, incluyendo las materias primas. La estructuración temporal observada a partir del análisis de instrumentos soporta lo observado en otras líneas de evidencia, como el registro faunístico e isotópico sobre restos humanos que sugiere cambios en el rol de los distintos recursos a lo largo del tiempo (Favier Dubois et al. 2009). En cuanto a la evidencia artefactual, Cardillo y Favier Dubois (2011) observaron una correlación entre la composición instrumental y los valores isotópicos que permitiría suponer un patrón temporal, lo cual concuerda con los resultados aquí expuestos

En trabajos anteriores (Cardillo \& Scartascini 2007; Alberti 2010) ha sido propuesto que la diferencia mencionada en el uso de las materias primas estaría relacionada con cambios en la movilidad de los grupos a lo largo del tiempo y con estrategias que eviten el riesgo, el cual se habría incrementado en el bloque más tardío, debido al incremento de la aridez y a la reducción de recursos hídricos. En relación con la estructuración cronológica de la diversidad instrumental que hemos observado, este cambio en la manufactura de instrumentos podría estar en relación con un cambio en el uso de las materias primas a lo largo del tiempo. Mientras que en el bloque más temprano se prefirieron las rocas locales y se verifica, en general, una diversidad instrumental menor, en el bloque más tardío se incorporan en mayor frecuencia rocas no inmediatamente disponibles y aumenta el número de clases artefactuales presentes en los diferentes loci. Esto es acorde con los valores isotópicos sobre restos esqueletales humanos que indican la mayor intensidad del componente terrestre en el bloque más tardío, con la consecuente diversificación del instrumental lítico para su explotación (Favier Dubois et al. 2009). Esta es una línea de evidencia a explorar en trabajos futuros.

\section{AGRADECIMIENTOS}

Este trabajo fue realizado en el marco del PIP CONICET 112-200801-00756 (2009-2011) titulado Paleoambientes, uso del espacio y los recursos en la costa norte del golfo San Matías 
desde su poblamiento, dirigido por el Dr. Cristian Favier Dubois, y dentro de la Beca Doctoral Tipo I otorgada a uno de los autores. Al Dr. Cristian Favier Dubois por sus comentarios y sugerencias. Lo expresado en este trabajo es de nuestra exclusiva responsabilidad.

\section{BIBLIOGRAFÍA}

Alberti, J. (2012). Fuentes de rocas y uso de materias primas líticas en Bahía Final 6, costa norte del golfo San Matías (Río Negro, Argentina). Intersecciones en Antropología, 13, 237-249.

Anderson, M. J. (2004). CAP: a FORTRAN computer program for canonical analysis of principal coordinates. New Zealand: Department of Statistics, University of Auckland.

Anderson, M. J. \& Robinson, R. (2003). Generalised discriminant analysis based on distances. Australian and New Zealand Journal of Statistics, 45(3), 301-318.

Anderson, M. J. \& Willis, T. J. (2003). Canonical analysis of principal coordinates: a useful method of constrained ordination for ecology. Ecology, 84, 511-525.

Borella. F., Mariano, C. \& Favier Dubois, C. (2007). Procesos tafonómicos en restos humanos en superficie en la localidad arqueológica de Bajo de la Quinta, Golfo San Matías (Río Negro). En F. Morello, M. Martinic, A. Prieto y G. Bahamonde (Eds.), Arqueología de Fuego-Patagonia. Levantando piedras, desenterrando huesos... y develando arcanos (pp. 403-410). Punta Arenas: Ediciones CEQUA.

Cardillo, M. (2009). Variabilidad en la manufactura y diseño de artefactos en el área costera patagónica. Un enfoque integrador. Tesis Doctoral inédita, Facultad de Filosofía y Letras, Universidad de Buenos Aires.

Cardillo, M. \& Favier Dubois, C. (2011). Una aproximación al uso del espacio en la costa norte del Golfo San Matías (Río Negro, Argentina): relaciones entre la evidencia artefactual e isotópica. En A. Guiance (Comp.), Movilidad y Migraciones. Actas de las III Jornadas Interdisciplinarias de Historia y Ciencias Humanas (241-252). Buenos Aires: CONICET - IMHICIHU.

Cardillo, M., Favier Dubois, C., Scartascini, F. \& Lípari, L. (2007). Una aproximación a la diversidad en la tecnología lítica de la costa Norte del Golfo San Matías, Rio Negro, Argentina. En Tras las huellas de la materialidad. Actas del XVI Congreso Nacional de Arqueología Argentina (pp. 323-328). San Salvador de Jujuy: Universidad Nacional de Jujuy.
Cardillo, M. \& Scartascini, F. (2007). Tendencias observadas en las estrategias de explotación de recursos líticos en el Golfo de San Matías, provincia de Río Negro, Argentina. En F. Morello, M. Martinic, A. Prieto y G. Bahamonde (Eds.), Arqueología de Fuego-Patagonia. Levantando piedras, desenterrando huesos... y develando arcanos (pp. 117-127). Punta Arenas: Ediciones CEQUA.

Cardillo, M. y Scartascini, F. (2011). Diversidad artefactual y explotación de materias primas en la costa norpatagónica, golfo San Matías, Río Negro. En F. Borella y M. Cardillo (Comps.), Arqueología de pescadores y marisqueadores en Nordpatagonia. Descifrando un registro de más de 6.000 años (pp. 68-86). Buenos Aires: Editorial Dunken.

Charlin, J. (2005). Utilización de materias primas líticas en el campo volcánico Pali Aike (Pcia. de Santa Cruz, Argentina). Una primera aproximación a partir del análisis de núcleos. Werken, 7, 39-55.

Charlin, J. (2009). Estrategias de aprovisionamiento y utilización de las materias primas líticas en el campo volcánico Pali Aike (Prov. Santa Cruz, Argentina). Oxford: BAR International Series 1901, Archaeopress.

Favier Dubois, C. (2009). Valores de efecto reservorio marino para los últimos 5.000 años obtenidos en concheros de la costa atlántica norpatagónica (golfo San Matías, Argentina). Magallania, 37(2), 139-147.

Favier Dubois, C. \& Borella, F. (2007). Consideraciones acerca de los procesos de formación de concheros en la costa Norte del golfo San Matías (Río Negro, Argentina). Cazadores-recolectores del Cono sur 2, 151-165.

Favier Dubois, C., Borella, F., Manzi, L., Cardillo, M., Lanzellotti, S., Scartascini, F., Carolina, M.\& Borgez Vaz, E. (2008). Aproximación regional al registro arqueológico de la costa rionegrina. En I. Cruz y S. Caracotche (Eds.), Arqueología de la Costa Patagónica. Perspectivas para la conservación (pp. 50-68). Río Gallegos: Universidad Nacional de la Patagonia Austral.

Favier Dubois, C., Borella, F. \& Tykot, R. (2009). Explorando tendencias en el uso humano del espacio y los recursos en el litoral rionegriono (Argentina) durante el Holoceno medio y tardío. En M. Salemme, F. Santiago, M. Álvarez, E. Piana, M. Vázquez y E. Mansur (Eds.), Arqueología de la Patagonia - Una mirada desde el último confín (pp. 985-997). Ushuaia: Editorial Utopías.

Favier Dubois, C. \& Kokot, R. (2011). Changing scenarios in Bajo de la Quinta (San Matías Gulf, Northern Patagonia, Argentina): Impact of geomorphologic processes in subsistence and human use of coastal habitats. Quaternary International, 245(1), 103-110. 
Favier Dubois, C. \& F. Scartascini. (2012). Intensive fishery scenarios on the North Patagonian coast (Río Negro, Argentina) during the Mid-Holocene. Quaternary International, 256(4), 62-70.

Fiore, D. \& Borella, F. (2010). Geometrías delicadas. Diseños grabados en cáscaras de huevo de Rheidae recuperados en la costa norte del Golfo San Matías, Río Negro. Intersecciones en Antropología, 11, 277-293.

Hammer, O. \& Harper, D. (2006). Paleontological Data Analisis. Oxford: Blackwell Publishing.

Hammer, O. Harper, D. \& Ryan, R. (2001). PAST Paleontological Statistics software package for education and data analysis. Palaeontologica Electronica, 4(1), 9.

Kuhn, S. (2004). Upper Paleolithic raw material economies at Ücagizlı cave, Turkey. Journal of Anthropological Archaeology, 23, 431-448.

Legendre, P. \& Fortín, M. (1989). Spatial pattern and ecological analysis. Vegetatio, 80, 107-138.

Legendre, P. \& Gallagher, E. (2001). Ecologically meaningful transformations for ordination of species data. Oecologia, 129, 271-280.

Legendre, P. \& Legendre, L. (1998). Numerical ecology. 2nd English Edition. Amsterdam: Elsevier Science BV.

Leps, J. y Smilauer, P. (2003). Multivariate analysis of ecological data using CANOCO. Cambridge: Cambridge University Press.

Magurran, A. (2004). Measuring Biological Diversity. Oxford: Blackwell Publishing.
Manzi, L., Favier Dubois, C. \& Borella, F. (2009). Identificación de agentes perturbadores y estrategias tendientes a la conservación del patrimonio arqueológico en la costa del Golfo de San Matías, provincia de Río Negro. Intersecciones en Antropología, 10, 3-16.

Marani, H. \& Scartascini, F. (2011). Integrando datos zooarqueológicos e isotópicos para una discusión de la subsistencia en la costa norte del Golfo San Matías. En II Congreso de Zooarquelogía Argentina. Libro de resúmenes, (p. 54). Olavarría: Universidad del Centro de la Provincia de Buenos Aires.

Scartascini, F. (2010). Explotación de peces en la costa norte del golfo San Matías (Río Negro): cambios y continuidades en la subsistencia y uso del espacio costero. Tesis de Licenciatura inédita, Facultad de Filosofía y Letras, Universidad de Buenos Aires.

Scartascini, F., Charo, M. y Volpedo, A. (2009). Caracterización de las estrategias de obtención de recursos ícticos a partir del análisis de los otolitos. El caso de la costa norte del golfo San Matías (Río Negro). En M. Salemme, F. Santiago, M. Álvarez, E. Piana, M. Vázquez y E. Mansur (Eds.), Arqueología de la Patagonia - Una mirada desde el último confín (pp. 845-852). Ushuaia: Editorial Utopías.

Shott, M. (2010). Size-dependence in Assemblage Measures: Essentialism, Materialism, and 'SHE' Analysis in Archaeology. American Antiquity, 75, 886-906. 
